\title{
Editorial
}

\section{Flashback sur les hallucinogènes}

\section{Flashback on hallucinogenic substances}

Jean VIGNAU

Service d'Addictologie, CHRU de Lille, 57, boulevard de Metz - 59037 LILLE CEDEX

Une bêtise mécanique et incontrôlable me posséda. Répétitions sans signification. Des êtres larvaires passaient en cortège devant moi dans une brume bleue, d'aucuns me lançaient des cris moqueurs et obscènes (plus tard j'identifiai ces cris : coassements des grenouilles) - J'ai dû vomir six fois. A quatre pattes secoué et tordu par les spasmes et la nausée. J'entendis les haut-le-cour et les borborygmes, comme si j'étais quelqu'un d'autre. J'étais couché près d'une roche. Des heures et des heures.

Lettres du Yage (lettres destinées en 1953 à un jeune poète inconnu, Allen Ginsberg) - W. Burroughs

Sous les apparences de la simplicité étymologique, la notion de drogue hallucinogène est en fait difficile à définir avec précision. La modernité voit essentiellement dans les hallucinogènes des substances recherchées pour leur capacité à induire des hallucinations, principalement visuelles. Ces produits sont pour la plupart d'origine végétale (issus de cactus, d'autres plantes ou de champignons) et connues des cultures ancestrales (tradition chamanique), même si le plus puissant d'entre eux est de découverte récente et de nature semi-synthétique (diéthylamide de l'acide lysergique ou LSD25). D'emblée, il faut souligner que cette capacité à induire des hallucinations n'est pas spécifique des hallucinogènes. Dans le cadre d'un travail de thèse de fin d'études médicales, j'ai personnellement tenté de recenser de manière exhaustive l'ensemble des substances (indépendamment de leur appartenance ou non au codex) susceptibles d'induire des états psychotiques avec hallucinations et/ou délire'. A l'évidence, cette propriété est hautement ubiquitaire. Ce qui caractérise en propre les drogues hallucinogènes, est que les phénomènes hallucinatoires attendus surviennent pour des doses non toxiques. Ceci veut dire que les hallucinations ne doivent pas être annonciatrices ou symptomatiques d'un état de déséquilibre physiologique global, provoqué par une intoxication suraiguë ou par le sevrage d'une utilisation chronique. Cette condition exclut, par exemple, les psychostimulants (amphétamines, cocaïne) dont seul le surdosage, au delà d'un seuil variable selon les individus, s'accompagne d'un syndrome psychotique hallucinatoire de manière systématique. Exit également le groupe - numériquement très important - des drogues à effet anti-cholinergique, qui obscurcit la conscience (confusion mentale). Cela ne signifie pas pour autant que les hallucinogènes se limitent à induire de purs phénomènes perceptifs hallucinatoires. Il est admis qu'ils provoquent aussi des modifications importantes du fonctionnement cognitif et émotionnel : la perception du temps et de l'espace, le traitement par le cerveau des stimuli extérieurs ainsi que la tonalité émotionnelle enveloppant l'expérience hallucinogène sont sujets à des fluctuations importantes au gré du voyage intérieur ${ }^{2}$. En bref, les hallucinogènes sont, à proprement parler, «thaumaturgiques» (selon l'expression très juste de Louis Lewin, père de la psychopharmacologie moderne, à la fin du $19^{\text {ème }}$ siècle) : elles doivent permettre à celui qui les prend de jouir pleinement

\footnotetext{
' J. Vignau "Les pharmacopsychoses" Thèse de doctorat en médecine - Lille $1990 ; 180 \mathrm{pp}$

2 J.-P. Valla "L'expérience hallucinogène" Masson - Paris 1983 ; $220 p p$
} 
du spectacle de sa propre expérience hallucinatoire, sans perdre totalement le sens des réalités. Remarquons à ce propos, que le terme d'hallucinose, soulignant la conservation d'une certaine distance critique, serait donc plus approprié que celui d'hallucination pour décrire les effets caractéristiques des hallucinogènes. Autant dire que les hallucinogènes représentent une classe de substances aux délimitations subtiles, fondées sur une expérience sophistiquée qui met en scène la propre subjectivité de l'individu par une distorsion temporaire de son rapport au monde. C'est ce qu'avait compris le chercheur canadien Humphrey Osmond en proposant le terme «psychédélique» pour désigner cette expérience, cette «vision enrichissante et initiatrice de changements existentiels» lors de son discours introductif à l'Académie des Sciences de New York, en 1957. Qu'elle soit culturelle, religieuse, esthétique ou transcendantale, la porte d'entrée vers l'usage de telles drogues est plutôt réservée à un petit groupe d'initiés. Et l'on comprend aisément que l'usage d'hallucinogènes soit demeuré une pratique très minoritaire (Rapport TREND et SINTES 2002 de l'OFDT $^{3}$ ) depuis son arrivée bruyante sur la scène occidentale sous l'impulsion du mouvement hippie dans les années 60 . S'il est si difficile de définir les drogues hallucinogènes, c'est donc semble-t-il parce qu'elles se rapportent à une expérience limite et complexe, une sorte d'apologie élitiste de la subjectivité, préservant le lien avec le monde objectif, sans véritable folie ni confusion.

Mais il est une seconde raison à cette autolimitation du phénomène psychédélique. C'est que contrairement aux dépendances à l'alcool, à l'héroïne ou à la cocaïne, l'usage d'hallucinogènes semble dégagé de toute nécessité adaptative. Il est exceptionnel en effet que les hallucinogènes soient utilisés au quotidien dans le but de supporter une condition humaine accablante. Comme le souligne avec beaucoup de pertinence Marc Deveaux dans son argument pour le présent colloque, les substances hallucinogènes ne présentent qu'une très faible propension à induire une accoutumance ${ }^{4}$. L'addictologue que je suis confirme absolument l'absence quasi-totale de demande de soutien à l'arrêt des hallucinogènes parmi les patients s'adressant aux centres spécialisés en toxicomanie (rapport du Ministère 1998-2003 ${ }^{5}$ ). Ce constat permet de bien distinguer le champ d'une addictologie

\footnotetext{
${ }^{3}$ P.Y. Bello, A. Toufik, M. Gandihon, I. Giraudon, N. Bonnet "Tendances récentes et nouvelles drogues en France 2002" Tendances OFDT juillet 2003 (31); 6 pp

${ }^{4}$ Ceci est d'autant plus étrange qu'il a été montré que la plupart des hallucinogènes ont la capacité d'agir chimiquement sur les circuits habituellement tenus pour responsables de l'état d'addiction. Ainsi, le LSD25, la mescaline et les autres phényléthylamines stimulent la transmission dopaminergique dans les structures mésolimbiques modulant les circuits neuraux du plaisir (ou récompense).
}

clinique, branche de la psychiatrie s'intéressant aux stratégies adaptatives psycho-pharmacologiques et à leurs aléas (l'usage de drogues comme automédication, au sens large) et celui d'une toxicologie clinique, se penchant sur la dangerosité des substances (les drogues comme poisons).

C'est ici que se situe le point de rencontre le plus classique et le plus important entre psychiatrie générale et approche toxicologique des hallucinogènes et sur lequel je conclurai cet éditorial. Plus encore que le psychiatreaddictologue, c'est le psychiatre-urgentiste qui est concerné par le thème central du colloque, à savoir l'approche toxicologique analytique des hallucinogènes d'origine végétale. La mondialisation des échanges fait que la plus exotique des substances peut facilement être achetée et consommée par nos concitoyens. Savoir identifier et doser ces substances apporte bien entendu une précieuse possibilité d'expertise dans la démarche diagnostique du clinicien appelé à prendre en charge un patient halluciné. C'est le cas notamment des bouffées délirantes et autre formes de psychoses aiguës dans lesquelles la question de l'empoisonnement est souvent posée avec insistance par le patient ou sa famille comme rationalisation permettant d'éviter la cruelle évidence du diagnostic. L'éventualité d'une prise de substance est aussi une question que doit se poser le médecin, en particulier devant certains éléments cliniques. Parmi ceux-ci la conservation d'une relative conscience du caractère pathologique des hallucinations est d'une grande valeur malgré certains doutes émis régulièrement quant à sa relativité culturelle «eurocentrée». Comme je l'ai souligné précédemment, l'expérience hallucinogène s'apparente plus à l'hallucinose qu'à de véritables hallucinations telles que l'on peut en rencontrer dans la schizophrénie, notamment. Or, la validité du critère d'absence de critique du caractère pathologique des troubles repose sur les résultats d'études transculturelles conduites par l'OMS ${ }^{6}$ et n'a jamais été démentie malgré une abondante recherche ces dernières années. Il en ressort que l'absence de critique ou de conscience des troubles (lack of insight) est le trait le plus invariant de la schizophrénie sous sa forme chronique ou en poussée aiguë, et ce quelque soit le contexte culturel. Si l'on se cale sur la procédure «à la française» d'imputabilité d'effets indésirables à un médicament, le critère de l'insight est probablement d'une grande significativité pour estimer le score sémiologique d'imputabilité intrinsèque de phénomènes perceptifs à une drogue hallucinogène.

\footnotetext{
${ }^{5}$ C. Palle, C. Bernard, E. Sévenier, H. Morfini “CSST 1998-2001 Exploitation des rapports d'activité-type des Centres Spécialisés de Soins au Toxicomanes, 1998, 2001" Focus OFDT DGS octobre $2003 ; 53 \mathrm{pp}$

${ }^{\circ}$ W. Wilson, T.A. Ban, W. Guys "Flexible system criteria in chronic schizophrenia" 1986 (27) 259-65
} 